\title{
Survey on Gesture Recognition for Hand Image Postures
}

\author{
Rafiqul Zaman Khan ${ }^{1} \&$ Noor Adnan Ibraheem ${ }^{1}$ \\ ${ }^{1}$ Faculty of Science, Department of Computer Science, Aligarh Muslim University, Aligarh, India \\ Correspondence: Rafiqul Zaman Khan, Faculty of Science, Department of Computer Science, Aligarh Muslim \\ University, Aligarh, 202002, India. Tel: 91-955-710-4757. E-mail: rzkhan.cs@amu.ac.in
}

Received: January 11, 2012 Accepted: February 29, 2012 Online Published: May 1, 2012

doi:10.5539/cis.v5n3p110

URL: http://dx.doi.org/10.5539/cis.v5n3p110

\begin{abstract}
One of the attractive methods for providing natural human-computer interaction is the use of the hand as an input device rather than the cumbersome devices such as keyboards and mice, which need the user to be located in a specific location to use these devices. Since human hand is an articulated object, it is an open issue to discuss. The most important thing in hand gesture recognition system is the input features, and the selection of good features representation. This paper presents a review study on the hand postures and gesture recognition methods, which is considered to be a challenging problem in the human-computer interaction context and promising as well. Many applications and techniques were discussed here with the explanation of system recognition framework and its main phases.
\end{abstract}

Keywords: computer vision, hand posture, hand gesture, hand gesture recognition, human computer interaction (HCI), virtual reality

\section{Introduction}

Gestures considered as a natural way of communication among human, since it is a physical movement of hands, arms, or body which conveying meaningful information. Gesture recognition then, is the interpretation of that movement as semantically meanings command. Gesture recognition has been studied in widely topics, and has a wide range of applications such as recognizing of sign language, human computer interaction (HCI), robot control, machine vision, smart surveillance, visual environments manipulating, etc (Ibraheem, 2011).

Although the term hand gesture and hand posture refers to the same meaning but there are some differences between them. Hand posture can be defined as the static movement, holding the hand with specific pose is a posture, for example a victory sign, pointing, and thumbs up. While in a complex posture, the fingers could be bent at any angle. The gesture can be defined as a dynamic movement, such as waving good-bye (Pavlovic, 1997; Mitra, 2007). A complex gesture is one that includes three things as mentioned by Mitra (2007) which are finger movement, wrist movement and changes in the hand's position and orientation. Examples of this type of gesture are the American Sign Language signs (Pavlovic, 1997).

Present approaches can be divided into Vision Based approaches and Data-Glove approaches. Data-Glove based approaches need a cumbersome devices have to be wore by user which connect the device to the computer, and that would reduce the natural level of the user's interaction with the computer (Mitra, 2007), for more details about data glove approaches, a survey on data glove are available in (Dipietro, 2008). Vision-based approaches, deals with some properties such as texture and color (Mitra, 2007) for a gesture analyzing; while tracking devices cannot. The number of cameras used by those techniques can be different; the speed and latency; and the environment structure, such as lighting condition or movement speed restrictions (Mitra, 2007). Examples of vision-based approaches are mentioned in (Zhu, 1998; Porta, 2002). Figure 1 shows an example of vision based and data glove based system. 


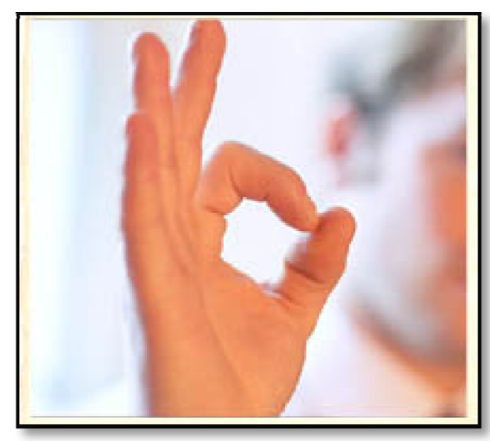

a

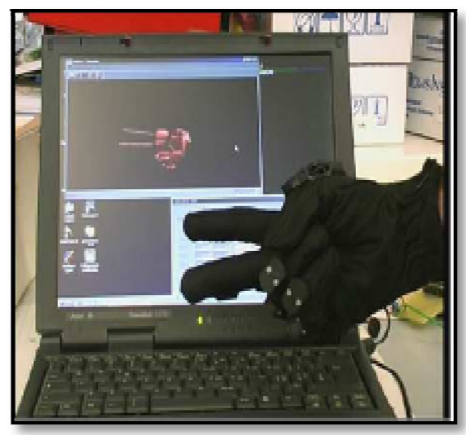

b

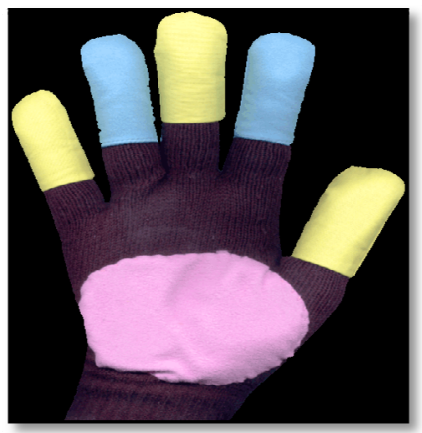

$\mathrm{c}$

Figure 1. Examples of data glove based and vision based approaches

a. vision based (from image gallery); b. data-glove based (Dipietro, 2008); c. colored glove (Lamberti, 2011).

Min (1997) classified hand gesture recognition system into several categories. Pavlovic (1997) presented a psychological aspects of gestures. A slightly updated version of this classification is given in Table 1.

Table 1. Hand gesture recognition system classification

\begin{tabular}{ll}
\hline Category & Type \\
\hline Application & Sign Language, Robot Control, Tracking Gesture, Games \\
Motion & $\begin{array}{l}\text { Static, Dynamic } \\
\text { Camera(s), Video, Data Glove Instrumented Device, } \\
\text { Colored Glove }\end{array}$ \\
Image acquired data & 2D, 3D \\
Data dimensions & One hand, two hands \\
number of hands used & 3D Hand Model, Appearance Based, Low Level Features \\
Input features & Communicative, Manipulative \\
\hline
\end{tabular}

Although other surveys have been done with various subsets of hand posture and gesture recognition (Pavlovic, 1997; Moeslund, 2001; Erol, 2007), this work is related to the vision based approaches and is up-to-date, and representing a good starting point for investigators interested in the field of hand postures and gestures as well.

The organization of this paper is as follows: Section 2 demonstrates approaches for hand posture and gesture recognition. Application areas for hand posture and gesture recognition are given in Section 3. Section 4 explains recognition system methodology. Conclusion of this paper is explained in Section 5.

\section{Approaches for Hand Posture and Gesture Recognition}

Gesture system can be one of the following three states, glove based, vision based and low level features based (Murthy, 2009), the vision based represents the most promising and effective alternative for glove based approaches that depends on sensors and wires which considered costly. The vision based needs camera(s) attached to a robot, and the gesture recognition algorithm responsible for translating the human gestures into a command to be carried out by the machine or robot (Hasan, 2011a).

A. 3D Hand Model based Approaches: Many methods have already been applied to analysis, model, and represent the hand shape, which gives a copious description and make a wide range of human hand to be represented, and a large database for storing the extracted shape characteristics is needed as well. Since 3D hand model has many DOFs besides the hand is an articulated deformable object, features extraction process became more difficult and formed an obstacle with already existing problems with 3D model based approach (Bilal, 2011). 
B. Appearance based approaches: Also known as View Based Approaches, which model the hand using the intensity of 2D images and define the gestures as a sequence of views. Appearance based approaches considered easier than3D model approaches, which led many researchers to search for alternative representations of the hand (Murthy, 2009; Hasan, 2011a).

C. Low Level Features based Approaches: Some gesture applications required a mapping between the input video and the gesture. Many researchers considered the full hand reconstruction is not essential for gesture system (Murthy, 2009; Hasan, 2011a). An example of low level features is, some geometric features which can extracted quickly and considered robust to noise (Murthy, 2009). Low-level features include: the centroid of the hand region (Pavlovic, 1997), an elliptical bounding region of the hand, edges, regions (Pavlovic, 1997), silhouettes (Erol, 2007), moments, and histograms (Pavlovic, 1997; Bilal, 2011).

\section{Application Areas for Hand Postures and Gestures}

Various applications have been used for hand postures and gestures as alternative level of interaction in different application domains, as mentioned in (Mitra, 2007): including virtual environments, smart surveillance, sign language translation, medical systems etc. This section gives a brief overview of some gesture recognition application areas (Mitra, 2007; Joseph, 1999). Hardware decreasing and reducing the processing cost can play a major factor for making the gesture recognition system the future setting and can offer more practical areas as well (Garg, 2009). Table 2 demonstrates some applications on gesture recognition system.

\subsection{Sign Language Recognition}

Sign language considered as an important and interesting application fields of hand posture and gesture recognition system (Joseph, 1999), where many systems have been applied for this purpose (Joseph, 1999). Sign language has special importance for communications since the gestures are the way used for interpretation and explanation of specific subject (Murthy, 2009). It can be used for disabled peopled when communicating with the other people, and with the computer as well (Murthy, 2009). American Sign Language in (Vogler, 2001; Starner, 2002) is one example that has received significant attention in the gesture literature. Kim (1996) recognized Korean Sign Language (KSL). Cho (2006) define a new gesture recognition algorithm for Korean scripts. Liang (1998) introduced lexicon of 250 vocabularies in Taiwanese Sign Language (TWL), Maraqa (2008) recognized Arabic Sign Language (ArSL). Murakami (1999) recognized Japanese sign language alphabets and words, they could recognize 42 alphabets and 10 words using two types of Neural Networks.

\subsection{Robotics, Human Manipulation and Instruction}

One of the effective applications that can utilize hand postures and gestures is robot tele-manipulation (Joseph, 1999). Telerobotic applications are typically classified under space exploration and military research domain (Murthy, 2009). Using gestures for controlling robots is corresponding to virtual reality interaction system (Murthy, 2009). Recent researches used postures and gestures to learn the robot some interaction commands by explaining its appropriate meaning for the robot as an action (Joseph, 1999). Various researches for robot control applications are implemented in (Malima, 2006; Wang, 2003; Bertsch, 2009).

\subsection{Virtual Reality}

For virtual reality application gestures have considered as one of the effective spreading stages in computing area (Murthy, 2009). Virtual reality interaction uses the hand gesture to manipulate the virtual movements using one or two hands for 2D and 3D interactions display (Murthy, 2009). Some virtual reality applications are available in (Bertsch, 2009; Rodriguez, 2008; Guan, 2008). Thomas (2008) worked with multimodal user interfaces that include visual, acoustic and haptic I/O. Based on the Wii controller hardware.

\subsection{Gesture-to-speech}

Gesture-to-speech application which converts hand gestures into speech, this system enables hearing-impaired people to communicate with their surrounding environments through computers and interacts easily with other people even without the knowing for the sign language (Joseph, 1999). Fels (1993) and Fels (1998) introduced Glove Talk system interface between speech synthesizer using data glove device which mapping hand-gestures to speech using neural networks.

\subsection{Games}

For computer games, $\mathrm{Xu}(2009)$ applied gesture recognition on virtual game applications. Chambers (2002) used hierarchical recognition of human gestures for sports video annotation. Rautaray (2010) implemented computer vision and gesture recognition techniques, and developed a vision based low cost input device for controlling the VLC player through gestures. 


\subsection{Television Control}

Last application for hand postures and gestures is controlling Television devices (Joseph, 1999). Freeman (1995) developed a system to control a television set by hand gestures. Using an open hand and the user can change the channel, turn the television on and off, increase and decrease the volume, and mute the sound.

Table 2. Applications on gesture recognition system

Reference
Applicaiton
(Yin, 2007) Humanoid service robot HARO-1; b. virtual robot.

(Rautaray, 2010)


(Freeman, 1995)

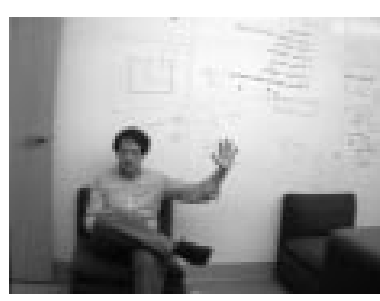

a

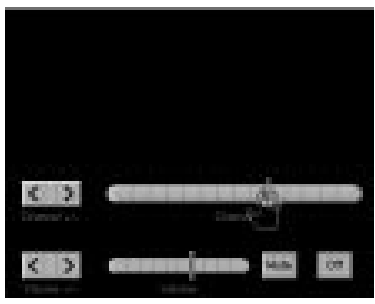

b

Hand gestures for television control

a. user has moved his hand for changing channel; b. channel changed.

\section{Recognition System Methodology}

Many researches have been suggested on gesture recognition system for different applications, with different recognition phases but they all agree with the main structure of the gesture recognition system. These phases are segmentation, features detection and extraction, and finally the classification or recognition phase. One of these structures illustrated in Figure 2.



Figure 2. The flow of gesture recognition system (Moni, 2009)

\subsection{Segmentation}

Segmentation phase plays an important role in the system recognition process. Perfect segmentation effects on the accuracy of the recognition system (Hasan, 2010). For any segmentation process, some image processing operations are required for hand shape detection (Murthy, 2009; Hasan, 2010).

Segmentation image algorithms can be classified into two types according to image gray level properties as explained in (Peter, 2011):

A. Discontinuity: Which tries to find a mass change in the contrast.

B. Similarity: Which computes the similarity between neighbor pixels. 
When the input gesture acquired form colored camera, instrumented glove device or colored glove as shown in Figure 1. The first step is segmentation, to extract the hand region from the input image and isolate it from the background (Hasan, 2010). There are two main methods for object segmentation, first method depends on the color model that can be extracted from the existence RGB color model which could be HSV color model (Hasan, 2010; Hasan, 2011a ; Mo, 2011) or YCbCr color space (Stergiopoulou, 2009); which deals with the pigment of the skin of the human hand (Hasan, 2010), the significant property of this color space is that the human different ethics group can be recognized according to their pigment concentration which can be distinguished according to some skin color saturation (Hasan, 2010). Then, the hand area is isolated from the input gesture with some threshold value. Some normalization for the segmented image might require for obtaining the gestures database which should be invariant against different perturbations like translation, scaling and rotation (Hasan, 2010). The database created with many samples per single gesture, the relation between the number of samples and the accuracy is directly proportional, and between number of samples and the speed is inversely proportional (Hasan, 2010).

Hasan (2010) used HSV color model to extract the skin-like hand region by estimating the parameter values for skin pigment, and used Laplacian filter for detection the edges. Stergiopoulou (2009) used YCbCr color model to segment the hand. Maraqa (2008) used color glove for input gestures and HSI color space for the segmentation process. Ghobadi (2008) treated the segmentation process as clustering method by grouping the image pixels among image objects. Lamberti (2011) used HSI color model to segment the hand object. Table 3 shows some applications of the segmentation methods used in the discussed method.

Table 3. Segmentation process from different hand gesture recognition methods

Reference

a. input image; b. segmented image; c. edge detection.

(Stergiopoulou, 2009)

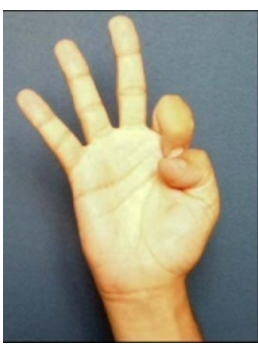

a

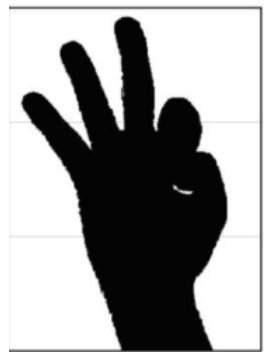

b
$\mathrm{YCbCr}$ color model used to segment the hand.

Hand segmentation

a. original image; b. segmented hand. 
(Maraqa, 2008)

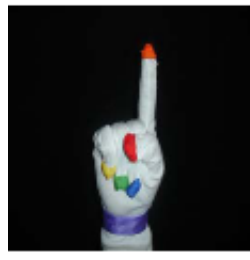

a

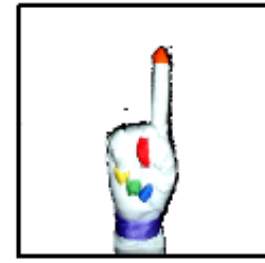

b

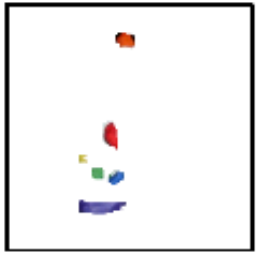

c
HSI color space used for the segmentation process.

Color image segmentation process

a. input hand glove image; b. segmented hand; c. segmented colors of the glove.

(Ghobadi, 2008)

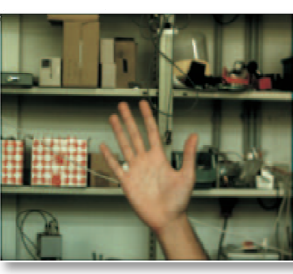

a

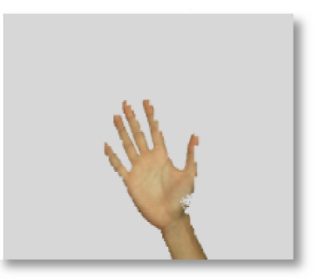

b
Clustering method used for segmentation by grouping the image pixels among image objects.

Hand segmentation

a. input image; b. segmented image.

(Li, 2003)

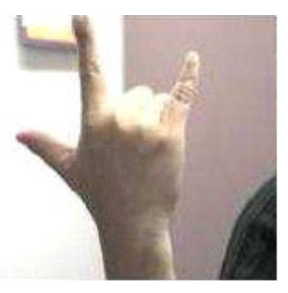

a

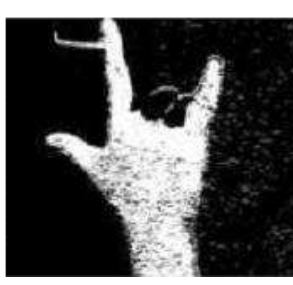

b
Transformed the image from RGB to HSV space and segment the hand.

Hand segmentation

a. input image; b. segmented image.

(Lamberti, 2011)

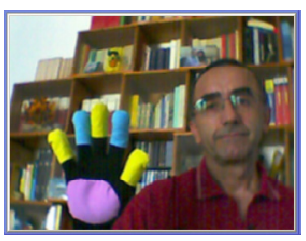

a

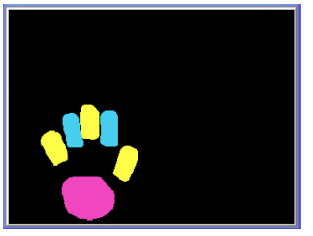

b
Used HSI color space to segment the hand region.

Hand segmentation

a. original image; b. image after segmentation. 


\subsection{Features Detection and Extraction}

The features are the useful information that can be extracted from the segmented hand object by which the machine can understand the meaning of that posture. The numerical representation of these features can be obtained from the vision perspective of the segmented hand object which form the feature extraction phase (Hasan, 2011b). Many researches have been applied to form this feature vector which takes different sizes as well as meanings. Hasan (2010) extracted the features vector by dividing the segmented hand object into fixed block size $5 \times 5$ brightness value moments; this produce 625 features vector size and only 98 are stored as actual features vector. Stergiopoulou (2009) applied Self-Growing and Self-Organized Neural Gas (SGONG) network to extract the exact shape of the hand region and determine three characteristics as the features; Palm region, Palm center, and Hand slope. Compute angle between the finger root and the hand center named RC Angle, and the joints the fingertip and the hand center named TC, and angle and distance from the palm center. Li (2003) defined a grid of fixed size with 12 blocks gray scale features vector, and each grid cell represents the mean value of the average brightness of the pixels in the block. Lamberti (2011) defined the distance $d$ from the palm to the fingers $d_{i}(\mathrm{i}=1, \ldots, 5)$, and computed the angle $\beta$ between the line connecting the centroids of the palm and the fingers, which produces four angles $\beta_{i}(\mathrm{i}=1, \ldots, 4)$, so the hand represented by nine numerical features vector (Lamberti, 2011). Table 4 demonstrates features vector representation of these methods.

Table 4. Features representation from different hand gesture recognition methods

Reference

a

Feature Vector representation

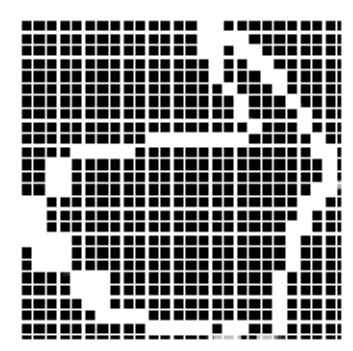

b

Description

By dividing the segmented hand object into fixed block size $5 \times 5$ geometric moments which is brightness value of each block separately.

a. segmented hand; b. features brightness division.

(Stergiopoulou, 2009)

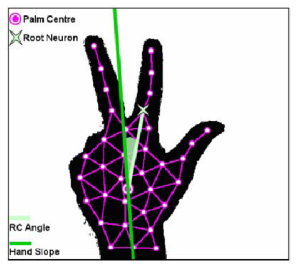

a

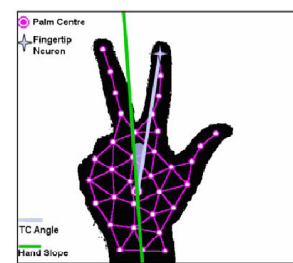

b

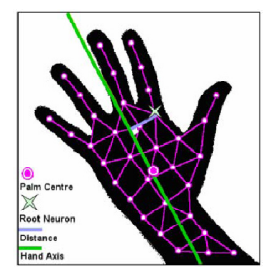

C
Feature Vector representation

a. RC angle; b. TC angle; c. distance from the palm center.

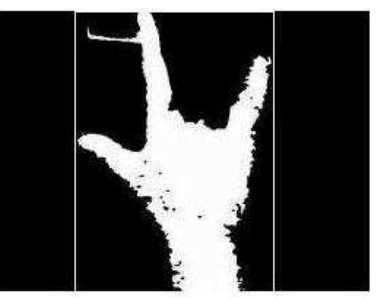

a
Feature vector

a. normalized hand; b. gray scale image partitioned into 12 blocks feature vector.
(Li, 2003)

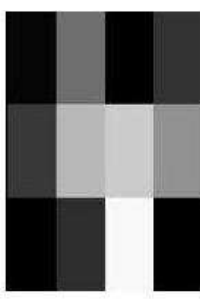

b
After extracted the hand shape using (SGONG) Neural Network algorithm. Three characteristics are determined; Palm region, Palm center, and Hand slope. value of the average
Defined a grid of fixed size with 12 blocks gray scale features vector, and each grid cell represents the mean brightness of the pixels in the segmented normalized image 


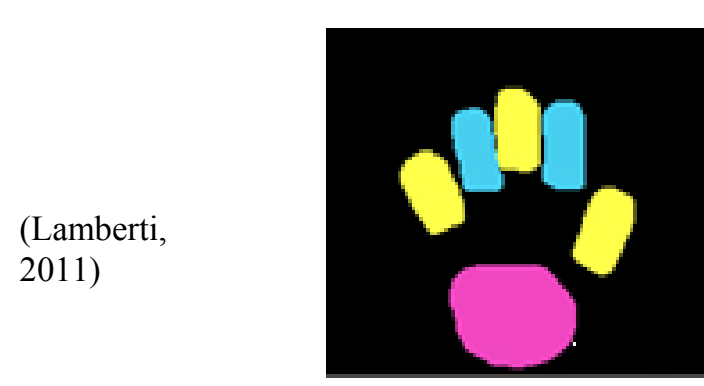

a

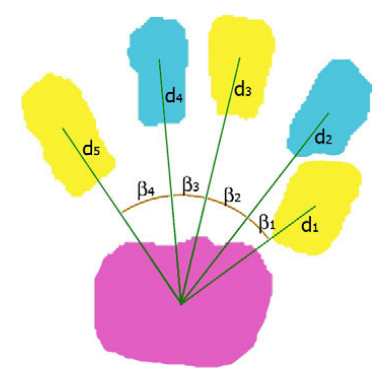

b
The features vector formed by five distances from palm to all fingers and four angles between those distances. (Lamberti, 2011)

Features representation.

a. segmented hand object; b. feature vector

\subsection{Recognition}

Recognition or classification of hand gestures is the last phase of the recognition system. Hand gestures can be classified using two approaches as mentioned in (Murthy, 2009).

A. Rule based Approaches: which represents the input features as manually encoded rule, and the winner gesture is the one that matched with the encoded rules after his features has been extracted. The main problem of this technique is that the human ability to encode the rules limits the successfulness of the recognition process (Murthy, 2009).

B. Machine Learning based Approaches: the most common approaches that considered the gesture as result of some stochastic processes (Murthy, 2009). Most of the problems that based on machine learning have been addressed based on the statistical modeling (Pavlovic, 1997), such as PCA (Kim, 2008), FSM (Verma, 2009). Hidden Markov Models (HMMs) (Keskin, 2003) have been paid attention by many researchers (Murthy, 2009), Kalman filtering (Mo, 2011), Artificial Neural networks (ANNs) (Maraqa, 2008; Murakami, 1999; Fels, 1993; Fels, 1998) which have been utilized in gesture recognition as well. Some researchers used Gaussian distribution for gestures classification (Stergiopoulou, 2009), and Euclidian distance metric (Hasan, 2010).

\section{Conclusion}

Human-machine interaction can be achieved by efficient gesture recognition system in which its applications varied from sign language recognition to games and virtual reality interfaces. In this paper a literature review on gesture recognition has been reviewed and analyzed; the major tools for classification process include FSM, PCA, HMMs, and ANNs are discussed. Descriptions of recognition system framework also presented with a demonstration of the main three phases of the recognition system by detection the hand, extraction the features, and recognition the gersture. The major image preprocessing steps necessarily required to features extraction phase are segmentation, edge detection, noise removing, and normalization, these steps may not applied together depending on targeted application.

\section{References}

Bertsch, F. A., \& Hafner, V. V. (2009). Real-time dynamic visual gesture recognition in human-robot interaction. IEEE-RAS 9th International Conference on Humanoid Robots (pp. 447-453). Paris. http://dx.doi.org/10.1109/ICHR.2009.5379541

Bilal, S., Akmeliawati, R., El, S. M. J., \& Shafie, A. A. (2011). Vision-based hand posture detection and recognition for sign language - A study. IEEE 4th international conference on Mechatronics Proceedings, (ICOM 2011), pp. 1-6. http://dx.doi.org/10.1109/ICOM.2011.5937178

Chambers, G. S., Venkatesh, S., West, G. A. W., \& Bui, H. H. (2002). Hierarchical Recognition of Intentional Human Gestures for Sports Video Annotation. IEEE 16th International Conference on Pattern Recognition Proceedings, 2, pp. 1082- 1085,http://dx.doi.org/10.1109/ICPR.2002.1048493

Cho., M. G. (2006). New gesture recognition algorithm and segmentation method of Korean scripts for gesture-allowed Ink editor. Elsevier an International Journal Information Sciences, 176(9), 1290-1303, http://dx.doi.org/10.1016/j.ins.2005.04.006 
Dipietro, L., Sabatini, A. M., \& Dario, P. (2008). Survey of glove-based systems and their applications. IEEE Transactions on systems, Man and Cybernetics, Part C: Applications and reviews, 38(4), 461-482. http://dx.doi.org/10.1109/TSMCC.2008.923862

Erol, A., Bebis, G., Nicolescu, M., Boyle, R. D., \& Twombly, X. (2007). Vision-based hand pose estimation: A review. Elsevier Computer Vision and Image Understanding, 108(1-2), 52-73. http://dx.doi.org/10.1016/j.cviu.2006.10.012

Fels, S. S., \& Hinton, G. E. (1993). Glove-talk: A neural network interface between a data-glove and a speech synthesizer. IEEE transaction on Neural Networks, 4(1), 2-8. http://dx.doi.org/10.1109/72.182690

Fels, S. S., \& Hinton, G. E. (1998). Glove-talk II-A neural-network interface which maps gestures to parallel formant speech synthesizer controls. IEEE transactions on neural networks, 9(1), 205-212. http://dx.doi.org/10.1109/72.655042

Freeman, W. T., \& Weissman, C. D. (1995). Television control by hand gestures. IEEE International Workshop on Automatic Face and Gesture Recognition. Zurich. Retrieved from www.merl.com/papers/docs/TR94-24.pdf

Garg, P., Aggarwal, N., \& Sofat, S. (2009). Vision based hand gesture recognition. World Academy of Science, Engineering and Technology. Retrieved from www.waset.org/journals/waset/v49/v49-173.pdf

Ghobadi, S. E., Loepprich, O. E., Ahmadov, F., Bernshausen, J., Hartmann, K., \& Lo®eld, O. (2008). Real time hand based robot control using multimodal images. International Journal of Copmuter Science IJCS.Vol 35(4). Retrieved from www.iaeng.org/IJCS/issues_v35/issue_4/IJCS_35_4_08.pdf

Guan, Y., \& Zheng, M. (2008). Real-time 3D pointing gesture recognition for natural HCI. IEEE Proceedings of the 7th World Congress on Intelligent Control and Automation WCICA, pp. 2 (Fels, 1998) 3 - 2 (Fels, 1998) 6. http://dx.doi.org/10.1109/WCICA.2008.4593304

Hasan, M. M., \& Mishra, P. K. (2010). HSV brightness factor matching for gesture recognition system. International Journal of Image Processing (IJIP), vol. 4(5), Retrieved from www.cscjournals.org/csc/manuscript/Journals/IJIP/.../IJIP-250.pdf

Hasan, M. M., \& Mishra, P. K. (2011a). Gesture recognition using modified HSV segmentation. IEEE International Conference on Communication Systems and Network Technologies. http://dx.doi.org/10.1109/CSNT.2011.75

Hasan, M. M., \& Mishra, P. K. (2011b). Brightness factor matching for gesture recognition system using scaled normalization. International Journal of Computer Science \& Information Technology (IJCSIT), 3(2). http://dx.doi.org/10.5121/ijcsit.2011.3203

Ibraheem, N. A., \& Khan, R. A. (2011). Survey on various gesture recognition technologies and techniques. Department of Computer Science, Aligarh Muslim University, Aligarh, India.

Joseph, J., \& LaViola, J. (1999). A survey of hand posture and gesture recognition techniques and technology. Master Thesis, NSF Science and Technology Center for Computer Graphics and Scientific Visualization, USA. Retrieved from ftp://ftp.cs.brown.edu/pub/techreports/99/cs99-11.ps.Z

Keskin, C., Erkan, A., \& Akarun, L. (2003). Real time hand tracking and 3d gesture recognition for interactive interfaces using HMM. Retrieved from www.cs.nyu.edu/ naz/docs/icann.pdfSimilar

Kim, J., \& Song, M. (2008). Three dimensional gesture recognition using PCA of stereo images and modified matching algorithm. IEEE Fifth International Conference on Fuzzy Systems and Knowledge Discovery, FSKD '08, pp. 116 - 120, Jinan Shandong. http://dx.doi.org/10.1109/FSKD.2008.610

Kim, J., Jang, W., \& Bien, Z. (1996). A dynamic gesture recognition system for the Korean sign language (KSL). IEEE transactions on systems, man, and cybernetics-part B: Cybernetics, 26(2), $354-359$. http://dx.doi.org/10.1109/3477.485888

Lamberti, L., \& Camastra, F. (2011). Real-time hand gesture recognition using a color glove. Springer-Verlag Berlin Heidelberg. ICIAP 2011, Part I, LNCS 6978, pp. 365-373. Retrieved from www.springerlink.com/index/02774HP03V0U4587.pdf

Li, X. (2003). Gesture recognition based on fuzzy C-Means clustering algorithm. Retrieved from citeseerx.ist.psu.edu/viewdoc/download?doi=10.1.1.136.3935.pdf 
Liang, R., \& Ouhyoung, M. (1998). A real-time continuous gesture recognition system for sign language. IEEE Third International Conference on Automatic Face and Gesture Recognition Proceedings, pp. 558 - 567. http://dx.doi.org/10.1109/AFGR.1998.671007

Malima, A., Özgür, E., \& Çetin, M. (2006). A fast algorithm for vision-based hand gesture recognition for robot control. IEEE $14^{\text {th }}$ conference on Signal Processing and Communications Applications, pp. 1- 4. http://dx.doi.org/10.1109/SIU.2006.1659822

Maraqa, M., \& Abu-Zaiter, R. (2008). Recognition of Arabic Sign Language (ArSL) using recurrent neural networks. IEEE First International Conference on the Applications of Digital Information and Web Technologies, ICADIWT 2008, pp. 478-48. http://dx.doi.org/10.1109/ICADIWT.2008.466(Fels, 1998)96

Min, B., Yoon, H., Soh, J., Yangc, Y., \& Ejima, T. (1997). Hand gesture recogrution using hidden markov models. IEEE International Conference on computional cybernetics and simulation. vol 5 . http://dx.doi.org/10.1109/ICSMC.1997.637364

Mitra, S., \& Acharya, T. (2007). Gesture recognition: A survey. IEEE Transactions on systems. Man and Cybernetics,Part C: Applications and reviews, 37(3), 311-324. doi:10.1109/TSMCC.2007.893280

Mo, S., Cheng, S., \& Xing, X. (2011). Hand gesture segmentation based on improved kalman filter and TSL skin color model. International Conference on Multimedia Technology (ICMT), Hangzhou. http://dx.doi.org/10.1109/ICMT.2011.6002133

Moeslund, T. B., \& Granum, E. (2001). A survey of computer vision-based human motion capture. Elsevier, Computer Vision and Image Understanding 81 - Modeling people toward vision-based understanding of a person's shape, appearance, and movement, 81(3), 231-268. http://dx.doi.org/10.1006/cviu.2000.0897

Moni, M. A. \& Ali, A. B. M. S. (2009). HMM based hand gesture recognition: A review on techniques and approaches. 2nd IEEE International Conference on Computer Scienceand Information Technology, (ICCSIT 2009). http://dx.doi.org/10.1109/ICCSIT.2009.5234536

Murakami, K., \& Taguchi, H. (1999). Gesture recognition using recurrent neural networks. ACM, Proceedings of the SIGCHI conference on Human factors in computing systems: Reaching through technologyCHI '91, pp. 237-242. http://dx.doi.org/10.1145/108844.108900

Murthy, G. R. S., \& Jadon, R. S. (2009). A review of vision based hand gestures recognition. International Journal of Information Technology and Knowledge Management, 2(2), 405-410. Retrieved from http://www.csjournals.com/IJITKM/PDF/34-G.R.S.Murthy_R.S.Jadon.pdf

Pavlovic, V. I., Sharma, R., \& Huang, T. S. (1997). Visual interpretation of hand gestures for human-computer interaction: A review. IEEE Transactions On Pattern Analysis And Machine Intelligence, 19(7), 677- 695. http://dx.doi.org/10.1109/34.598226

Peter, H. P. (2011). Image Segmentation.(1sted.). India. (Part 2). Image Segmentation Methods Image Segmentation through Clustering Based on Natural Computing Techniques. Retrieved from www.scribd.com/doc/70993072/Image-Segmentation

Porta, M. (2002). Vision-based user interfaces: Methods and applications. Elsevier, International Journal Human-Computer Studies,2002(57), 27-73. http://dx.doi.org/10.1006/ijhc.1012

Rautaray, S. S., \& Agrawal, A. (2010). A vision based hand gesture interface for controlling VLC media player. International Journal of Computer Applications, 10(7). Retrieved from www.ijcaonline.org/volume10/number7/pxc3872012.pdf

Rodriguez, O. P., Gonzalez, O. O., Avizzano, C. A., Ruffaldi, E., Vercelli, D., \& Bergamasco, M. (2008). Development of a 3D real time gesture recognition methodology for virtual environment control. IEEE Proceedings 17th International Symposium on Robot and Human Interactive Communication, pp. 279-284. http://dx.doi.org/10.1109/ROMAN.2008.4600679

Starner, T., Weaver, J., \& Pentland, A. (2002). Real-time American Sign Language recognition using desk and wearable computer based video. IEEE Transactions onPattern Analysis and Machine Intelligence, 20(12), 1371 - 1375. http://dx.doi.org/10.1109/34.735811

Stergiopoulou, E., \& Papamarkos, N. (2009). Hand gesture recognition using a neural network shape fitting technique. Elsevier Engineering Applications of Artificial Intelligence 22, 1141-1158. http://dx.doi.org/10.1016/j.engappai.2009.03.008 
Thomas, S., Benjamin, P., Niels, H., \& Susanne, B. (2008). Gesture recognition with a Wii controller. ACM, Proceedings of the Second International Conference on Tangible and Embedded Interaction (TEI'08), Germany. http://dx.doi.org/10.1145/1347390.1347395

Verma, R., \& Dev, A. (2009). Vision based hand gesture recognition using finite state machines and fuzzy logic. IEEE International Conference onUltra Modern Telecommunications \& Workshops, ICUMT '09, pp. 1-6, Petersburg. http://dx.doi.org/10.1109/ICUMT.2009.5345425

Vogler, C., \& Metaxas, D. (2001). A framework for recognizing the simultaneous aspects of American Sign Language. Ideal Computer Vision and Image Understanding 81, pp. 358-384. http://dx.doi.org/10.1006/cviu.2000.0895

Wang, H. H. X., Mandal, M. K., Meng, M., \& Li, D. (2003). Efficient face and gesture recognition techniques for robot control. IEEE Canadian Conference on Electrical and Computer Engineering, CCECE 2003, 3, 1757-1762. http://dx.doi.org/10.1109/CCECE.2003.1226250

Xu, Z., Xiang, C., Lantz, V., \& Kong-qiao, W. (2009). Hand gesture recognition and virtual game control based on 3D accelerometer and EMG sensors. ACM Proceedings of the 14th international conference on Intelligent user interfaces IUI '09. http://dx.doi.org/doi:10.1145/1502650.1502708

Yin, X., \& Xie, M. (2007). Hand posture segmentation, Recognition and Application for Human-Robot Interaction. Retrieved from www.intechopen.com/download/pdf/pdfs_id/5276

Zhu, Y., Huang, Y., Xu, G., Ren, H., \& Wcn, Z. (1998). Vision-based Interpretation of Hand Gestures by Modeling Appearance Changes in Image Sequences. WA '98 IAPH Workshop on Machine Vision Applications.Makuhm. Japan. Retrieved from www.mva-org.jp/Proceedings/.../1998/papers/1998573.pdf 